\title{
Tardives prises de conscience
}

\author{
Jean Martin
}

Dr méd., membre de la rédaction

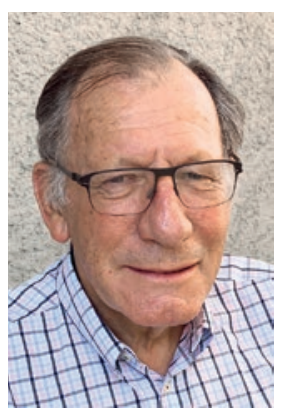

Les mouvements d'opinion des derniers mois concernant le racisme et les relations femmes-hommes m'interpellent, en tant qu'homme et en tant que médecin; et je pense n'être pas seul à reconsidérer des attitudes que je croyais adéquates.

Sur le premier sujet: ma famille et moi avons vécu dans les deux Amériques, en Inde, puis en Afrique (huit ans en tout) et y avons développé des amitiés durables. De retour au pays, j'ai continué à être très «interculturel»; j'ai présidé durant neuf ans la section vaudoise de la Ligue contre le racisme et l'antisémitisme et fait partie de comités d'organisations de développement. Ma conviction née de l'expérience: "La différence, c'est toujours intéressant, laissons-nous enrichir par les atouts de la diversité.»

\section{«C'est l'évidence: ces formes d'infériorité» sont systémiques et perdurent.»}

De notre séjour aux Etats-Unis, je garde un bon souvenir de l'intégration des Afro-américains - en milieu universitaire. Mais la force et l'étendue des manifestations qui ont suivi l'homicide de George Floyd à Minneapolis, ainsi que les témoignages multiples de personnes d'ascendance africaine (entre autres), aux USA, en Suisse et ailleurs, m'ont vivement questionné. Suis-je resté aveugle, au cours des décennies? N'ai-je pas saisi ce que ressentent dans leur quotidien nos concitoyens «différents» devant trop d'attitudes qui vont de la maladresse à l'inadmissible: réserve, malaise, évitement, rejet voire gestes et paroles insultantes? Au travail, à l'école, dans la rue, dans les loisirs? J'ai cru trop vite que le problème était réglé pour l'essentiel, que les réflexes racistes étaient devenus très minoritaires. Le très persistant racisme ordinaire remis en lumière me bouscule. On ne lynche pas ici de jeunes Noirs, mais que dire de quantité d'attitudes stigmatisantes qui font sentir qu'ils/elles ne sont "pas comme nous» et pas bienvenus?

Même examen de conscience pour les rapports femme-homme. Je pense avoir été correct, courtois et aidant avec mes collaboratrices et collègues - et j'ai cru que les choses se passaient raisonnablement dans ce pays. Même si je savais, sans attendre les coups de projecteur de \#MeToo, que beaucoup de femmes avaient à souffrir de paroles et gestes à connotation sexuelle (j'ai eu à m'en préoccuper en tant que médecin cantonal). Les dernières Marches des femmes du 14 juin m'ont fait mieux saisir l'acuité et la gravité durables du problème.

Il faut se rendre à l'évidence: ces formes d'«infériorité» - respectivement de "supériorité» - sont systémiques et perdurent. Au bénéfice de l'homme à peau blanche, systématiquement favorisé, indépendamment de toute donnée personnelle ou circonstancielle. Toutes choses égales par ailleurs, cela reste en 2020 une muette loi d'airain.

Il y a des améliorations, mais éradiquera-t-on un jour complètement ces inégalités profondément inscrites? Si la promulgation de lois, chartes et déclarations est indispensable pour poser un cadre ferme, ces textes ne sauraient régler ce qui relève du fine tuning, de comportements usuels et quotidiens, "non scandaleux", qui gardent une dimension sexiste ou raciste. C'est à chacun-e d'en prendre conscience personnellement et d'évoluer.

On pourra juger ces propos excessifs, «autoflagellation à la suisse», voire atteinte à la liberté d'expression. Débat classique. Je crois à cette formule: «La liberté de chacun ne vaut que par les limites qu'on lui met.» Un mot sur les appels, dans la foulée de Black Lives Matter, à déboulonner statues ou plaques honorant des personnages qui ont fait fortune dans la traite des êtres humains (pour parfois se montrer généreux dans leur lieu d'origine). N'espérons pas trouver un catalogue de principes permettant de trancher chaque cas avec justice. On n'efface pas l'histoire, mais on peut l'analyser.

\section{«Ces différences de traitement restent en 2020 une muette loi d'airain.»}

L'important est que les enfants à l'école, et la collectivité, soient informés de manière factuelle et équilibrée; et que soient menés des débats sur les «différences» alléguées entre personnes et groupes: pourquoi les uns continueraient-ils à être jugés "moins égaux que les autres»? Qu'est-ce qui fait perdurer ce phénomène? Beau défi pédagogique.

Notre société est confrontée à d'autres enjeux de survie, apparemment plus lourds encore. Pourtant, les inégalités et les atteintes à la dignité charriées par «Le Système» (et notre inconscient?) sont un immense enjeu. 